\title{
The Probabilities of Obtaining Solitary Wave and Other Solutions in the Modified Noguchi Power Line
}

\author{
Jean R. Bogning ${ }^{1}$, Cédric Jeatsa Dongmo ${ }^{2}$ and Clément Tchawoua ${ }^{3}$ \\ ${ }^{1}$ University of Bamenda, Higher Teacher Training College Bambili, Department of Physics, Bamenda, Cameroon \\ ${ }^{2}$ University of Yaoundé 1, Faculty of Science, Laboratory of Mechanics, Yaoundé, Cameroon \\ Correspondence: Jean R. Bogning, University of Bamenda, Bamenda, Cameroon
}

Received: February 28, 2021 Accepted: May 24, $2021 \quad$ Online Published: June 25, 2021

doi:10.5539/jmr.v13n4p19 URL: https://doi.org/10.5539/jmr.v13n4p19

\begin{abstract}
We use the implicit Bogning function (iB-function) to proceed to a kind of inventory of the possible solutions of the modified nonlinear partial differential equation which characterizes the modified power line of Noguchi. Firstly, we make an inventory of the forms of solutions through a field of possible solutions, then we identify the most probable forms that we set out to look for. The iB-function is used because it summarizes within it several types of different functions depending on the choice of its characteristics and it is easy to handle in the case of strongly nonlinear partial differential equations. In other words, we use the notion of probability to locate, through the characteristic indices of iB-functions, the forms of solitary and traveling wave solutions likely to propagate in the modified Noguchi power line.
\end{abstract}

Keywords: power line, iB-functions, solitary wave, range equations, field, possibilities, solutions

\section{Introduction}

Physics is defined simply as the science which deals with and studies bodies in motion. It is closely linked to other sciences and in particular Mathematics and numerical calculations to name just the most used, to analyze and study the feasibility of the physical system considered. It is also subdivided into several study segments in which the dynamics of nonlinear excitations in power lines occupy an important place (Tiague Takongmo \& Bogning, 2018; Tiague Takongmo \& Bogning, 2018; Tiague Takongmo \& Bogning, 2018; Tiague Takongmo \& Bogning, 2018; Tiague Takongmo \& Bogning, 2018; Tiague Takongmo \& Bogning, 2018).Speaking of the power line, the modified one from Noguchi (Tiague Takongmo \& Bogning, 2018) is a bit peculiar in its construction. It is made of a set of identical cells made up of coils and capacitors. The nonlinear nature of the line is maintained by charge capacitors which vary in a non-linear manner. Other forms of forced solutions have already been proposed in the context of some research work, but what we bring is the inventory and the possibilities of obtaining the majority or all forms of solutions including new forms of solutions and among others, solitary waves, periodic waves and even certain ordinary solutions through the probabilities of obtaining each type of solution (Ngouo Tchinda \& Bogning, 2020).

We remain here in our dynamic of proposing the solutions and the techniques of resolution of the so-called complicated nonlinear partial differential equations (Bogning et al., 2012; Bogning et al., 2012; Bogning et al., 2013; Bogning et al., 2013; Bogning et al., 2015; Bogning, 2015, Bogning, 2015; Bogning et al., 2016; Djeumen Tchaho et al., 2012; Djeumen Tchaho et al., 2011; Djeumen Tchaho et al., 2010, Bogning et al., 2015; Bogning et al., 2013; Njikue et al., 2018; Bogning \&Kofané, 2006; Bogning \&Kofané, 2006; Bogning, 2018; Njikué et al., 2018; Sarwar, S.\& Rashidi MM. 2016 ). Mainly, within the framework of this work, the iB-functions (Bogning J. R. (2019). Mantématique: Les fonctions implicites de Bogning \&applications. Germany, EUE.; Bogning J. R. (2019). Mathematics for physics: The implicit Bogning functions \& Applications. Germany, LAB.; Bogning J. R. (2019). Mathematics for nonlinear physics: Solitary wave in the center of the resolution of dispersive nonlinear partial differential equations. USA, Dorrance Publishing Co.; Bogning J. R. (2020). Eléments de la mécanique analytique et de la Physique quantique. Germany, EUE.; Bogning J. R. (2020). Elements of Analytical Mechanics and Quantum physics. Germany, LAB.) are used to find and build these solutions, precisely because of the flexibility in the most complicated calculations.

Certainly, as mentioned a little above, apart from our work, several works have dealt with the resolution of nonlinear partial differential equations, but the particularity of this article is that it draws its originality from the works that we have been developing for a while and we are continuing to develop them. The use of the notion of probability to locate and construct solutions of nonlinear partial differential equations is quite new and follows a logical approach which takes its foundation in our previous work. It is not just a question for us of solving the equations, while placing ourselves in a 
context in relation to many works produced by different authors, but pushing the limits to see how far we can get with the new approach used.

This manuscript is organized into five main sections, namely, the presentation of the model, some notions on iB-functions, forms of solutions and solving the equations and the review of the comments on the solutions obtained as conclusion.

\section{Noguchi Nonlinear Modified Power Line}

Noguchi's modified electric line is constituted by a number $n$ of identical cells where the capacitors of constant capacitance $C$ are connected in parallel across the terminals of the coils of constant inductance $L$ both connected to a capacitor of variable charge and crossed by the voltage $u_{n}$.

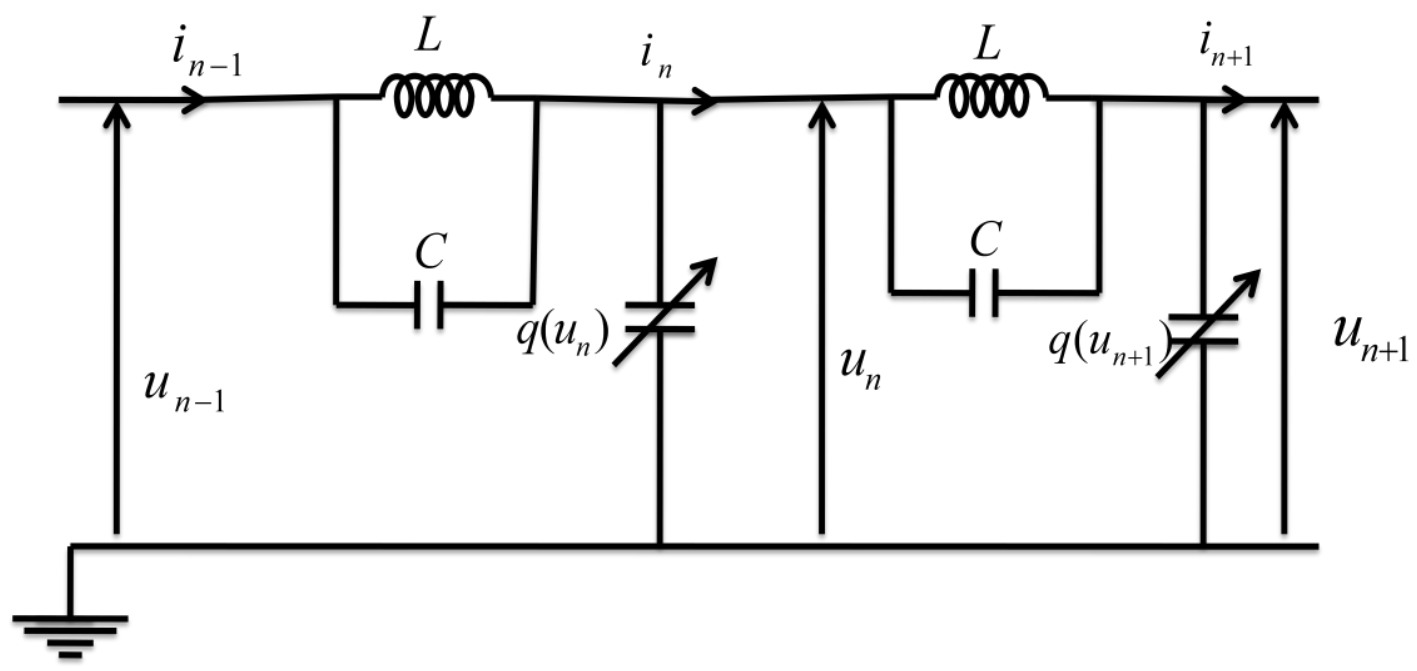

Figure 1. Presentation of a modified non-linear Noguchi electrical line

The equation which describes the dynamics of signal propagation in this power line has been established [7] and given by

$$
n_{1} U_{x x x x}+n_{2} U_{x x}+n_{3} U_{t t x x x}+n_{4} U_{t t x x}-L[q(U(x, t))]=0,
$$

where $U(x, t)$ represents the signal or voltage in the circuit, $n_{1}=h^{4} / 12, n_{2}=h^{2}, n_{3}=L C h^{4} / 12, n_{4}=L C h^{2}$ and $h$ the step between the cells that make up the network, the nonlinear charge of the capacitor is given by

$$
[q(U(x, t))]=a_{1} U(x, t)+a_{2} U^{3}(x, t)+a_{3} U^{5}(x, t),
$$

where $a_{i}(i=1,2,3)$ are non zero constants.

Equation (1) is the generalized form of the nonlinear partial differential equation which describes the propagation dynamics in the Noguchi power line.

\section{3. iB-functions}

iB- implicit functions are generally defined by

$$
J_{n, m}\left(\sum_{i=0}^{p} \alpha_{i} x_{i}\right)=\sinh ^{m}\left(\sum_{i=0}^{p} \alpha_{i} x_{i}\right) / \cosh ^{n}\left(\sum_{i=0}^{p} \alpha_{i} x_{i}\right),
$$

where $J_{n, m}\left(\sum_{i=0}^{p} \alpha_{i} x_{i}\right)$ represents the implicit form of the function, $\sinh ^{m}\left(\sum_{i=0}^{p} \alpha_{i} x_{i}\right) / \cosh ^{n}\left(\sum_{i=0}^{p} \alpha_{i} x_{i}\right)$ the explicit form of the function, $\alpha_{i}(i=0,1,2, \ldots, p)$ represent the parameters associated with the independent variables $x_{i}$ $(i=0,1,2, \ldots, p)$, the pair $(n, m) \in R^{2}$ indicates the power of the function. More precisely, $n$ is the power of $\cosh \left(\sum_{i=0}^{p} \alpha_{i} x_{i}\right)$ and $m$ the power of $\sinh \left(\sum_{i=0}^{p} \alpha_{i} x_{i}\right)$. This function, as defined in relation (1), is also called the iB-functions of several variables and any derivative operation undertaken in this case is partial.

The iB-functions of a single variable is defined by 


$$
J_{n, m}(\alpha x)=\sinh ^{m}(\alpha x) / \cosh ^{n}(\alpha x),
$$

Where $J_{n, m}(\alpha x)$ represents the implicit form of the function, $\alpha$ represents the parameter associated with the independent variable $x$, the pair $(n, m) \in R^{2}$ indicates the power of the function.

Some important transformations are given by

$$
\begin{gathered}
J_{n+1, m+1}=J_{n, m} J_{1,1}, \\
J_{n+2, m+2}=J_{n, m} J_{2,2}, \\
J_{n+p, m+p}=J_{n, m} J_{p, p}, \\
J_{n-p, m-p}=J_{n, m} J_{-p,-p}, \\
J_{n+p, m+p}=J_{2 p, 2 p} J_{n-p, m-p}, \\
J_{n, m} . J_{n^{\prime}, m^{\prime}}=J_{n+n^{\prime}, m+m^{\prime}}, \\
J_{n, m} . J_{m, n}=J_{m+n, m+n}, \\
J_{m_{1}, n_{1}} . J_{m_{2}, n_{2}} \ldots J_{m_{p}, n_{p}}=J_{m_{1}+m_{2}+\ldots+m_{p}, n_{1}+n_{2}+\ldots+n_{p}}, \\
\frac{d J_{n, m}}{d x}=m \alpha J_{n-1, m-1}-n \alpha J_{n+1, m+1}
\end{gathered}
$$

Some of properties in its compact forms which facilitate the addition and multiplication of expressions are given by the following formulas

$$
J_{n, m}(x+y)=\frac{\sum_{k^{\prime}=0}^{m} C_{m}^{k^{\prime}} J_{-k^{\prime}, m-k^{\prime}}(x) J_{-m+k^{\prime}, k^{\prime}}(y)}{\sum_{k=0}^{n} C_{n}^{k} J_{-n+k, k}(x) J_{-n+k, k}(y)},
$$

By matching $y \rightarrow-y$, we obtain

$$
J_{n, m}(x-y)=\frac{\sum_{k^{\prime}=0}^{m}(-1)^{k^{\prime}} C_{m}^{k^{\prime}} J_{-k^{\prime}, m-k^{\prime}}(x) J_{-m+k^{\prime}, k^{\prime}}(y)}{\sum_{k=0}^{n}(-1)^{k} C_{n}^{k} J_{-n+k, k}(x) J_{-n+k, k}(y)},
$$

The compact trigonometric formulas which result from formulas (14) and (15) are given by

$$
J_{n, m}[i(x+y)]=\frac{\sum_{k^{\prime}=0}^{m} C_{m}^{k^{\prime}} J_{-k^{\prime}, m-k^{\prime}}(i x) J_{-m+k^{\prime}, k^{\prime}}(i y)}{\sum_{k=0}^{n} C_{n}^{k} J_{-n+k, k}(i x) J_{-n+k, k}(i y)}, i^{2}=-1,
$$

and

$$
J_{n, m}[i(x-y)]=\frac{\sum_{k^{\prime}=0}^{m}(-1)^{k^{\prime}} C_{m}^{k^{\prime}} J_{-k^{\prime}, m-k^{\prime}}(i x) J_{-m+k^{\prime}, k^{\prime}}(i y)}{\sum_{k=0}^{n}(-1)^{k} C_{n}^{k} J_{-n+k, k}(i x) J_{-n+k, k}(i y)}, i^{2}=-1 .
$$




\section{Forms of Solutions and Solving the Equation}

We seek the solutions of equation (1) in the form

$$
U(x, t)=a J_{n, m}\left(\alpha x-\alpha_{0} t\right),
$$

where $a, \alpha, \alpha_{0}$ are arbitrary non zero constants. Under these conditions, we transform equation (1) by posing the change of variable $\xi=\alpha x-\alpha_{0} t$. So, we get at first

$$
\left(n_{1} \alpha^{4}+n_{4} \alpha_{0}^{2} \alpha^{2}\right) U_{\xi \xi \xi \xi}+n_{2} \alpha^{2} U_{\xi \xi}+n_{3} \alpha^{4} \alpha_{0}^{4} U_{\xi \xi \xi \xi \xi \xi}-L[q(U(\xi))]=0
$$

with

$$
q(U(\xi))=a_{1} U(\xi)+a_{2} U^{3}(\xi)+a_{3} U^{5}(\xi) .
$$

Taking into account equation (19) in (18) allows to obtain

$$
\left(n_{1} \alpha^{4}+n_{4} \alpha_{0}^{2} \alpha^{2}\right) U_{\xi \xi \xi \xi}+\left(n_{2} \alpha^{2}-L \alpha_{0}^{2} a_{1}\right) U_{\xi \xi}+n_{3} \alpha^{4} \alpha_{0}^{4} U_{\xi \xi \xi \xi \xi \xi}-L \alpha_{0}^{2} a_{2}\left(U^{3}\right)_{\xi \xi}-L \alpha_{0}^{2} a_{3}\left(U^{5}\right)_{\xi \xi}=0,
$$

Equation (22) is the one that will be the object of the effective study in this work. Taking into account the transformation $\xi=\alpha x-\alpha_{0} t$, the sought solution becomes as follows

$$
U(\xi)=a J_{n, m}(\xi)
$$

\subsection{Main Equation of Range of Coefficients}

Taking into account the ansatz (23) in the equation (22) imposes the calculation of its different terms. for this purpose we obtain after successive calculations, the derivative of order 5 of $\xi=\alpha x-\alpha_{0} t$ given by

$$
U_{\xi \xi \xi \xi \xi}=\alpha_{1} J_{n-5, m-5}-\alpha_{2} J_{n-3, m-3}+\alpha_{3} J_{n-1, m-1}-\alpha_{4} J_{n+1, m+1}+\alpha_{5} J_{n+3, m+3}-\alpha_{6} J_{n+5, m+5},
$$

where $\alpha_{i}(i=1,2, \ldots, 6)$ are the function of $n, m$ and given by

$$
\begin{gathered}
\alpha_{1}=a m(m-1)(m-2)(m-3)(m-4), \\
\alpha_{2}=a m(m-1)(m-2)(m-3)(n-4)+a m(m-1)(m-2)^{2}(n-3) \\
+a m(m-1)(n-2)(m-1)(m-2)+a m^{2}(n-1)(m-2)^{2}+a m n(m+1)(m-1)(m-2), \\
\alpha_{3}=a m(m-1)(m-2)(n-3)(n-2)+a m(m-1)(n-2)^{2}(m-1) \\
+a m^{2}(n-1)(m-2)(n-2)+a m n(m+1)(m-1)(n-2)+a m^{2}(m-1)(n-2)(n-1) \\
+a m^{3}(n-1)^{2}+a m^{2} n(m+1)(n-1)+a n m^{2}(n-1)(m+1)+a m n^{2}(m+1)^{2}+a n m(n+1)(m+2)(m+1), \\
\alpha_{4}=a m(m-1)(n-2)(n-1) n+a m^{2}(n-1)^{2} n+a m n^{2}(m+1)(n-1) \\
+a n^{2} m(n-1)(m+1)+a n^{3}(m+1)^{2}+a n^{2}(n+1)(m+2)(m+1)+a n m(n-1)(n+1)(m+2) \\
+a n^{2}(m+1)(n+1)(m+2)+a n(n+1)^{2}(m+2)^{2}+a n(n+1)(n+2)(m+3)(m+2), \\
\alpha_{5}=a n m(n-1)(n+1)(n+2)+a n^{2}(m+1)(n+1)(n+2)+a n(n+1)^{2}(m+2)(n+2) \\
+a n(n+1)(n+2)^{2}(m+3)+a n(n+1)(n+2)(n+3)(m+4),
\end{gathered}
$$

and

$$
\alpha_{6}=\operatorname{an}(n+1)(n+2)(n+3)(n+4) \text {. }
$$

In the same logic of the calculations of the successive derivatives of $U$ with respect to $\xi$ we have 


$$
\begin{aligned}
& U_{\xi \xi \xi \xi \xi \xi}=\alpha_{1}(m-5) J_{n-6, m-6}-\left[\alpha_{1}(n-5)+\alpha_{2}(m-3)\right] J_{n-4, m-4}+\left[\alpha_{2}(n-3)+\alpha_{3}(m-1)\right] J_{n-2, m-2} \\
& -\left[\alpha_{3}(n-1)+\alpha_{4}(m+1)\right] J_{n, m}+\left[\alpha_{4}(n+1)+\alpha_{5}(m+3)\right] J_{n+2, m+2}-\left[\alpha_{5}(n+3)+\alpha_{6}(m+5)\right] J_{n+4, m+4} \\
& +\alpha_{6}(n+5) J_{n+6, m+6} .
\end{aligned}
$$

The introduction of the different derivatives in equation (22) leads to the following main coefficient range equation

$$
\begin{aligned}
& A_{1} J_{n-4, m-4}+A_{2} J_{n-2, m-2}+A_{3} J_{n, m}+A_{4} J_{n+2, m+2}+A_{5} J_{n+4, m+4}+A_{6} J_{n-6, m-6}+A_{7} J_{n+6, m+6} \\
& -A_{8} J_{3 n-2,3 m-2}+A_{9} J_{3 n, 3 m}-A_{10} J_{3 n+2,3 m+2}-A_{11} J_{5 n-2,5 m-2}+A_{12} J_{5 n, 5 m}-A_{13} J_{5 n+2,5 m+2}=0,
\end{aligned}
$$

where $A_{i}(i=1,2, \ldots, 13)$ are given by

$$
\begin{aligned}
& A_{1}=a\left(n_{1} \alpha^{4}+n_{4} \alpha_{0}^{2} \alpha^{2}\right)(m-1)(m-2)(m-3)-n_{3} \alpha_{0}^{4} \alpha^{4}\left[\alpha_{1}(n-5)+\alpha_{2}(m-3)\right], \\
& A_{2}=\left(n_{1} \alpha^{4}+n_{4} \alpha_{0}^{2} \alpha^{2}\right)\left[\begin{array}{l}
a m(m-1)(m-2)(n-3)+\operatorname{am}(m-1)(n-2)(m-1) \\
+\operatorname{am}^{2}(n-1)(m-2)+\operatorname{amn}(m+1)(m-1)
\end{array}\right] \\
& +a m(m-1)\left(n_{2} \alpha^{2}-L \alpha_{0}^{2} a_{1}\right)+n_{3} \alpha_{0}^{4} \alpha^{4}\left[\alpha_{2}(n-3)+\alpha_{3}(m-1)\right] \text {, } \\
& A_{3}=\left(n_{1} \alpha^{4}+n_{4} \alpha_{0}^{2} \alpha^{2}\right)\left[\begin{array}{l}
\operatorname{am}(m-1)(n-2)(n-1)+a m^{2}(n-1)^{2}+a m n(m+1)(n-1) \\
+\operatorname{anm}(n-1)(m+1)+a n^{2}(m+1)^{2}+a n(n+1)(m+2)(m+1)
\end{array}\right] \\
& -\left(n_{2} \alpha^{2}-L \alpha_{0}^{2} a_{1}\right)[a m(n-1)+a n(m+1)]-n_{3} \alpha_{0}^{4} \alpha^{4}\left[\alpha_{3}(n-1)+\alpha_{4}(m+1)\right], \\
& A_{4}=\left(n_{1} \alpha^{4}+n_{4} \alpha_{0}^{2} \alpha^{2}\right)\left[\begin{array}{l}
\operatorname{anm}(n-1)(n+1)+a n^{2}(m+1)(n+1) \\
+a n(n+1)^{2}(m+2)+\operatorname{an}(n+1)(n+2)(m+3)
\end{array}\right] \\
& -a\left(n_{2} \alpha^{2}-L \alpha_{0}^{2} a_{1}\right) n(n+1)-n_{3} \alpha_{0}^{4} \alpha^{4}\left[\alpha_{4}(n+1)+\alpha_{5}(m+3)\right] \text {, } \\
& A_{5}=a\left(n_{1} \alpha^{4}+n_{4} \alpha_{0}^{2} \alpha^{2}\right) n(n+1)(n+2)(n+3)-n_{3} \alpha_{0}^{4} \alpha^{4}\left[\alpha_{5}(n+3)+\alpha_{6}(m+5)\right] \text {, } \\
& A_{6}=n_{3} \alpha_{0}^{4} \alpha^{4} \alpha_{1}(m-5), \\
& A_{7}=n_{3} \alpha_{0}^{4} \alpha^{4} \alpha_{6}(n+5), \\
& A_{8}=3 a^{3} m(3 m-1) L \alpha_{0}^{2} a_{2}, \\
& A_{9}=L \alpha_{0}^{2} a_{2}\left[3 a^{3} m(3 n-1)+3 a^{3} n(3 m+1)\right], \\
& A_{10}=3 a^{3} L \alpha_{0}^{2} a_{2} n(3 n+1), \\
& A_{11}=5 a^{5} L \alpha_{0}^{2} a_{3} m(5 m-1), \\
& A_{12}=L \alpha_{0}^{2} a_{3}\left[5 a^{5} m(5 n-1)+5 a^{5} n(5 m+1)\right],
\end{aligned}
$$

and

$$
A_{13}=5 a^{5} L \alpha_{0}^{2} a_{3} n(5 n+1) .
$$




\subsection{Field of Possible Solutions}

In this section, we study the possibilities of grouping the terms of the equation (31) and finding solutions. Thus, to obtain the values of $n$ and $m$ for which certain terms of equation (31) regroup, we solve the pairs of equations in $n$ and $m$ such that if $A_{i} J_{n, m}$ and $A_{j} J_{n^{\prime}, m^{\prime}}$ (with $i \neq j$ ) are two terms of the equation (31), we have simultaneously $n=n^{\prime}$ and $m=m^{\prime}$.

In this exercise, we count a total of 51 pairs of equations which lead to the determination of the pairs $(n, m)$. So we have

$$
n, m \in\left\{-4,-3,-\frac{3}{2},-\frac{3}{2},-1,-\frac{1}{2}, 0, \frac{1}{2}, 1, \frac{3}{2}, 2,3,4\right\} .
$$

The combination of the values of $n$ and $m$ for which there is a grouping of the terms makes it possible to obtain a table of the possibilities of solutions comprising 169 pairs.

The 169 pairs constitute the extended field of pairs for which the search for solutions must be made. But the probabilities of appearance of pairs make it possible to determine the most probable couples and thereby reduce the field of possibilities in order to obtain a restricted field of possibilities in which the effective research will be carried out.

\subsection{Probabilities of Appearance of Pairs $(n, m)$ and Dominant Pairs}

To obtain the values of $n$ and $m$ for which certain terms of equation (30) regroup, we have solved 51 pairs of equations in $n$ and $m$. The probabilities of appearance of the pairs $(n, m)$ are given by $P(-1,-1)=8 / 51, P(-2,-2)=5 / 51$, $P(0,0)=9 / 51, P(-1 / 2,-1 / 2)=3 / 51, P(-3 / 2,-3 / 2)=2 / 51, P(2,2)=5 / 51, P(1,1)=8 / 51, P(1 / 2,1 / 2)=3 / 51$, $P(4,4)=1 / 51, P(3,3)=2 / 51, P(3 / 2,3 / 2)=2 / 51, P(-4,-4)=1 / 51, P(-3,-3)=2 / 51$.

With regard to the probabilities of appearance, the pairs $(0,0),(-1,-1)$ and $(1,1)$ are the dominant pairs. We can define the restricted field of possibilities by combining the values of $n$ and $m$ of the dominant pairs in the table below.

Table 1. Restricted field of possibilities

\begin{tabular}{|cccc|}
\hline$(n, m)$ & -1 & 0 & 1 \\
\hline-1 & $(-1,-1)$ & $(-1,0)$ & $(-1,1)$ \\
0 & $(0,-1)$ & $(0,0)$ & $(0,1)$ \\
1 & $(1,-1)$ & $(1,0)$ & $(1,1)$ \\
\hline
\end{tabular}

The goal of obtaining the restricted field of couples aims to verify whether in addition to the dominant couples, there are other couples which lead to non-trivial solutions. Thus, in the following lines, we will solve equation (32) for the different values of the above pairs.

\subsection{Solving the Secondary Equations From the Main Coefficient Range Equation}

In this section, we look for the solutions of equation (32) for the pairs of the restricted field of possibilities.

For the pairs $(0,0),(-1,1),(1,-1),(1,0),(0,-1)$ and $(0,1)$ the equation admits trivial solutions so that the real search for solutions is done only for the pairs $(-1,-1),(1,1)$ and $(-1,0)$.

$$
\text { * Case }(n, m)=(-1,-1)
$$

Taking into account the pair $(n, m)=(-1,-1)$ in equation (32) allows to write

$$
\left(A_{1}-A_{8}+A_{12}\right) J_{-5,-5}+\left(A_{2}+A_{9}-A_{13}\right) J_{-3,-3}+\left(A_{3}-A_{10}\right) J_{-1,-1}+\left(A_{6}-A_{11}\right) J_{-7,-7}=0 \text {. }
$$

Equation (47) holds if and only if we have

$$
\begin{gathered}
A_{1}-A_{8}+A_{12}=0, \\
A_{2}+A_{9}-A_{13}=0, \\
A_{3}-A_{10}=0,
\end{gathered}
$$

and

$$
A_{6}-A_{11}=0
$$


The calculations of $\alpha_{i}(i=1,2, \ldots, 6)$ and $A_{j}(j=1,2, \ldots, 13)$ for $(n, m)=(-1,-1)$ allow to rewrite the above equations for $a \neq 0$ as

$$
\begin{gathered}
12\left(n_{1} \alpha^{4}+n_{4} \alpha_{0}^{2} \alpha^{2}\right)+852 n_{3} \alpha_{0}^{4} \alpha^{4}-6 a^{2} L \alpha_{0}^{2} a_{2}+25 L \alpha_{0}^{2} a_{3} a^{4}=0, \\
-21\left(n_{1} \alpha^{4}+n_{4} \alpha_{0}^{2} \alpha^{2}\right)+\left(n_{2} \alpha^{2}-L \alpha_{0}^{2} a_{1}\right)+634 n_{3} \alpha_{0}^{4} \alpha^{4}+9 L \alpha_{0}^{2} a^{2}-10 L \alpha_{0}^{2} a_{3} a^{4}=0, \\
8\left(n_{1} \alpha^{4}+n_{4} \alpha_{0}^{2} \alpha^{2}\right)-\left(n_{2} \alpha^{2}-L \alpha_{0}^{2} a_{1}\right)-142 n_{3} \alpha_{0}^{4} \alpha^{4}-3 a^{2} L \alpha_{0}^{2} a_{2}=0, \\
24 n_{3} \alpha_{0}^{4} \alpha^{4}-a^{4} L \alpha_{0}^{2} a_{3}=0 .
\end{gathered}
$$

The equation (55) allows to determine

$$
a=\alpha\left(\frac{24 n_{3} \alpha_{0}^{2}}{L a_{3}}\right)^{\frac{1}{4}}, n_{3} a_{3} \succ 0 .
$$

The introduction of equation (56) in equations (52), (53) and (54) makes it possible to determine the constraint relations that the other parameters must verify. The solution under these conditions is given by

$$
U(\xi)=\alpha\left(\frac{24 n_{3} \alpha_{0}^{2}}{L a_{3}}\right)^{\frac{1}{4}} J_{-1,-1}(\xi) \Rightarrow U(x, t)=\alpha\left(\frac{24 n_{3} \alpha_{0}^{2}}{L a_{3}}\right)^{\frac{1}{4}} J_{-1,-1}\left(\alpha x-\alpha_{0} t\right)
$$

- Case $(n, m)=(1,1)$

Taking into account the pair $(n, m)=(1,1)$ in equation (31) allows to write

$$
\left(A_{3}-A_{8}\right) J_{1,1}+\left(A_{4}+A_{9}-A_{11}\right) J_{3,3}+\left(A_{5}-A_{10}+A_{12}\right) J_{5,5}+\left(A_{7}-A_{13}\right) J_{7,7}=0,
$$

Equation (58) holds if and only if we have

$$
\begin{gathered}
A_{3}-A_{8}=0, \\
A_{4}+A_{9}-A_{11}=0, \\
A_{5}-A_{10}+A_{12}=0,
\end{gathered}
$$

and

$$
A_{7}-A_{13}=0
$$

The calculations of $\alpha_{i}(i=1,2, \ldots, 6)$ and $A_{j}(j=1,2, \ldots, 13)$ for $(n, m)=(-1,-1)$ allow to rewrite the above equations for $a \neq 0$ as

$$
\begin{gathered}
8\left(n_{1} \alpha^{4}+n_{4} \alpha_{0}^{2} \alpha^{2}\right)-\left(n_{2} \alpha^{2}-L \alpha_{0}^{2} a_{1}\right)-136 n_{3} \alpha_{0}^{4} \alpha^{4}-3 a^{2} L \alpha_{0}^{2} a_{2}=0, \\
-20\left(n_{1} \alpha^{4}+n_{4} \alpha_{0}^{2} \alpha^{2}\right)-\left(n_{2} \alpha^{2}-L \alpha_{0}^{2} a_{1}\right)-616 n_{3} \alpha_{0}^{4} \alpha^{4}+9 a^{2} L \alpha_{0}^{2} a_{2}-10 L \alpha_{0}^{2} a_{3} a^{4}=0, \\
12\left(n_{1} \alpha^{4}+n_{4} \alpha_{0}^{2} \alpha^{2}\right)-840 n_{3} \alpha_{0}^{4} \alpha^{4}-6 a^{2} L \alpha_{0}^{2} a_{2}+25 a^{4} L \alpha_{0}^{2} a_{3}=0,
\end{gathered}
$$




$$
24 n_{3} \alpha_{0}^{4} \alpha^{4}-a^{4} L \alpha_{0}^{2} a_{3}=0 .
$$

Equation (66) also gives

$$
a=\alpha\left(\frac{24 n_{3} \alpha_{0}^{2}}{L a_{3}}\right)^{\frac{1}{4}}, n_{3} a_{3} \succ 0 .
$$

The constraint relations linked to the value of $a$ for this solution to be valid are obtained by introducing (67) in the equations (63), (64) and (65). The solution in this case is given by

$$
U(\xi)=\alpha\left(\frac{24 n_{3} \alpha_{0}^{2}}{L a_{3}}\right)^{\frac{1}{4}} J_{1,1}(\xi) \Rightarrow U(x, t)=\alpha\left(\frac{24 n_{3} \alpha_{0}^{2}}{L a_{3}}\right)^{\frac{1}{4}} J_{1,1}\left(\alpha x-\alpha_{0} t\right) .
$$

* Case $(n, m)=(-1,0)$

Taking into account the pair $(n, m)=(-1,0)$ in equation (32) allows to write

$$
A_{3} J_{-1,0}+A_{9} J_{-3,0}-A_{10} J_{-1,2}-A_{13} J_{-3,2}=0,
$$

Equation (67) holds if and only if for the values of $\alpha_{i}(i=1,2, \ldots, 6)$ and $A_{j}(j=1,2, \ldots, 13)$ and setting $(n, m)=(-1,0)$

we have

$$
\begin{gathered}
n_{1} \alpha^{4}+n_{2} \alpha^{2}+n_{4} \alpha_{0}^{2} \alpha^{2}-L \alpha_{0}^{2} a_{1}+n_{3} \alpha_{0}^{4} \alpha^{4}=0, \\
L \alpha_{0}^{2} a_{2}=0,
\end{gathered}
$$

and

$$
L \alpha_{0}^{2} a_{3}=0 \text {. }
$$

As we are not only in the logic of solving the nonlinear partial differential equation, but also in the logic of mathematically obtaining the partial differential equations which admit as solutions the forms of solutions considered from the start, then the disposition of the above equations impose to modify the structure of the partial differential equation which models the modified electric line of Noguchi. So, we set $a_{2}=a_{3}=0$ and equation (22) reduces to

$$
\left(n_{1} \alpha^{4}+n_{4} \alpha_{0}^{2} \alpha^{2}\right) U_{\xi \xi \xi \xi}-\left(n_{1} \alpha^{4}+n_{4} \alpha_{0}^{2} \alpha^{2}+n_{3} \alpha_{0}^{4} \alpha^{2}\right) U_{\xi \xi}+n_{3} \alpha^{4} \alpha_{0}^{4} U_{\xi \xi \xi \xi \xi \xi}=0,
$$

such that

$$
a_{1}=\frac{\alpha^{2}\left(n_{1} \alpha^{2}+n_{2}+n_{4} \alpha_{0}^{2}+n_{3} \alpha_{0}^{4} \alpha^{2}\right)}{L \alpha_{0}^{2}},
$$

as predict in equation (70). In this case the solution of equation (73) is given by

$$
U(\xi)=a J_{-1,0}(\xi) \Rightarrow U(x, t)=a J_{1,1}\left(\alpha x-\alpha_{0} t\right), a \in R .
$$

\section{Conclusion}

What motivated the design and the realization of this work is that for the resolution of the complicated partial differential equations or strongly nonlinear, it is often very difficult to obtain the solutions while passing by the simple integral methods. Under these conditions what remains to be done is to seek the so-called forced solutions. The forced solutions consist in considering a form of solution and that one imposes it as solution of nonlinear partial differential 
equation considered and thereafter one starts to look for the conditions or to fix the constraints so that this is possible.

Once these constraints and conditions are fixed, the so-called forced solution from the start becomes the exact or approximate solution, depending on the margin of error committed during the resolution. Animated by this logic, we wanted to check whether or not there are solutions that can propagate or not, in the modified Noguchi power line or simply the solutions which verify the nonlinear partial differential equation which govern the dynamics of propagation in the modified Noguchi power line. At this level of reflection, the choice of the ansatz or solution function becomes very important. As the iB-function has the advantage of wrapping within it several types of functions and in particular exponential, hyperbolic and circular functions, we have opted for the iB-function of power $(n, m)$. So the search for solutions to equation (22) revolves around the choice of pairs $(n, m)$ because each pair $(n, m)$ corresponds to a very precise function. However, generally $n$ and $m$ are reals and their arbitrary choices of which are very difficult and tedious to make. Another advantage of the use of the iB-function is that from the equation of ranges of coefficients obtained, we can determine the values of $(n, m)$ for which certain terms are grouped together. We note in the same sense that when there are several terms which regroup, the probability that the pairs $(n, m)$ which are at the origin of this regrouping lead to the solution function, is high. On the other hand, when the pair $(n, m)$ allows very little grouping, the number of chances for it to define the solution function is also very low.

Beyond this general view, the probabilities of grouping or merging the terms are such that the highest are $P(0,0)=9 / 51, P(-1,-1)=8 / 51$ and $P(1,1)=8 / 51$. Apart from the pair $(0,0)$ which leads to the trivial solution, we noticeed in most of the cases we have treated so far that the solutions obtained were foreseen by the dominant pairs which are in the case of this problem $(-1,-1)$ and $(1,1)$.

To return to physics, these solutions are solitary wave solutions of the kink type. The periodic traveling waves can also be obtained by choosing $\alpha$ and $\alpha_{0}$ pure imaginary complexes.

Beyond the work carried out in this manuscript, its physical and mathematical scope is to know in general, how to choose without too much hassle, the forms of progressive wave solutions or not of the nonlinear partial differential equations governing the dynamics of propagation in the waveguides. The concept of probability introduced will henceforth make it possible to locate, without much hesitation, the characteristics of the solitary wave solution-function or not likely to propagate in the waveguide considered.

\section{References}

Bogning, J. R. (2013). Analytical soliton solutions and wave solutions of discrete nonlinear cubic-quintique Ginzburg-Landau equations in array of dissipative optical system. American Journal of Computational and Applied Mathematics, 3(2), 97-105.

Bogning, J. R. (2013). Pulse soliton solutions of the modified KdV and Born-Infeld equations. International Journal of Modern Nonlinear Theory and Application, 2, 135-140. https://doi.org/10.4236/ijmnta.2013.22017

Bogning, J. R. (2015). Nth Order Pulse Solitary Wave Solution and Modulational Instability in the Boussinesq. Equation American Journal of Computational and Applied Mathematics, 5(6), 182-188.

Bogning, J. R. (2015). Sechn Solutions of the generalized and modified Rosenau-Hyman Equations. Asian Journal of Mathematics and Computer Research, 9(1), 2395-4205.

Bogning, J. R. (2018). Exact solitary wave solutions of the $(3+1)$ modified B-type Kadomtsev-Petviashvili family equations. Amer. J. Comput. Appl. Maths., 8, 85-92.

Bogning, J. R. (2019). Mathematics for nonlinear physics :Solitary wave in the center of the resolution of dispersive nonlinear partial differential equations. Dorrance Publishing Co, USA.

Bogning, J. R. (2019). Mathématics for physics: The implicit Bogning functions \& applications. Lambert Academic, Publishing, Germany.

Bogning, J. R. (2019). Mathématique: les fonctions implicites de Bogning \& Applications. Editions universitaires Européenne, Germany.

Bogning, J. R. (2020). Eléments de la Mécanique Analytique et de la Physique quantique. Editions universitaires Européenne, Germany.

Bogning, J. R. (2020). Elements of Analytical Mechanics and Quantum Physics. Lambert Academic, Publishing, Germany.

Bogning, J. R., \& Kofane, T. C. (2006). Analytical solutions of the discrete nonlinear Schrödinger equation in arrays of optical fibers. Chaos, Solitons \& Fractals, 28(1), 148-153. https://doi.org/10.1016/j.chaos.2005.04.121 
Bogning, J. R., \& Kofane, T. C. (2006). Multi-instability of gap solitons and dynamics of nonlinear excitations in the array of optical fibers. Chaos, Solitons \& Fractals, 27(2), 377-385. https://doi.org/10.1016/j.chaos.2005.04.023

Bogning, J. R., Djeumen Tchaho, C. T., \& Kofané, T. C. (2013). Solitary wave solutions of the modified Sasa-Satsuma nonlinear partial differential equation. American Journal of Computational and Applied Mathematics, 3(2), 97-107.

Bogning, J. R., Kuiaté, G. F., Omanda, H. M., \& Tchaho, C. D. (2015). Combined Peakons and multiple-peak solutions of the Camassa-Holm and modified KdV equations and their conditions of obtention. Physics Journal, 1(3), 367-374.

Bogning, J. R., Porsezian, K., Kuiaté, G. F., \& Omanda, H. M. (2015). gap solitary pulses induced by the Modulational instability and discrete effects in array of inhomogeneous optical fibers. Physics Journal, 1(3), 216-224. https://doi.org/10.9734/BJMCS/2016/10620

Bogning, J. R., Tchaho, C. D., \& Omanda, H. M. (2016). Combined solitary wave solutions in higher-order effects optical fibers. Journal of Advances in Mathematics and Computer Science, 1-12. https://doi.org/10.9734/BJMCS/2016/10620

Bogning, J. R., Tchaho, C. T. D., \& Kofané, T. C. (2012). Construction of the soliton solutions of the Ginzburg-Landau equations by the new Bogning-Djeumen Tchaho-Kofané method. Physica Scripta, 85(2), 025013. https://doi.org/10.1088/0031-8949/85/02/025013

Bogning, J. R., Tchaho, C. T. D., \& Kofané, T. C. (2012). Generalization of the Bogning-Djeumen Tchaho-Kofané method for the construction of the solitary waves and the survey of the instabilities. Far East Journal of Dynamical systems, 20(2), 101-119.

Djeumen Tchaho, C. T., Bogning, J. R., \& Kofane, T. C. (2010). Construction of the analytical solitary wave solutions of modified Kuramoto-Sivashinsky equation by the method of identification of coefficients of the hyperbolic functions. Far East J. Dyn. Sys., 14(1), 14-17.

Djeumen Tchaho, C. T., Bogning, J. R., \& Kofane, T. C. (2011). Multi-soliton solutions of the modified Kuramoto-Sivashinsky's equation by the BDK method. Far East J. Dyn. Sys., 15(2), 83-98.

Djeumen Tchaho, C. T., Bogning, J. R., \& Kofané, T. C. (2012). Modulated soliton solution of the modified Kuramoto-Sivashinsky's equation. American Journal of Computational and Applied Mathematics, 2(5), 218-224. https://doi.org/10.5923/j.ajcam.20120205.03

Guy, T. T., \& Bogning, J. R. (2018). Construction of solitary wave solutions of higher-order nonlinear partial differential equations modeled in a nonlinear hybrid electrical line. American Journal of circuits, systems and signal processing, 4(3), 36-44.

Guy, T. T., \& Bogning, J. R. (2018). Construction of solitary wave solutions of higher-order nonlinear partial differential equations modeled in a nonlinear capacitive electrical line. American Journal of circuits, systems and signal processing, 4(2), 15-22.

Guy, T. T., \& Bogning, J. R. (2018). Construction of Solitary Wave Solutions of Higher-Order Nonlinear Partial Differential Equations Modeled in a Modified Nonlinear Noguchi Electrical Line. American Journal of circuits, systems and signal processing, 4(1), 8-14. https://doi.org/10.9734/jamcs/2019/v33i230174

Guy, T. T., \& Bogning, J. R. (2018). Construction of solutions in the shape (Pulse; Pulse) and (Kink; Kink) of a set of two equations modeled in a nonlinear inductive electrical line with crosslink capacitor. American Journal of circuits, systems and signal processing, 4(2), 28-35.

Guy, T. T., \& Bogning, J. R. (2018). Coupled soliton solutions of modeled equations in a Noguchi electrical line with crosslink capacitor. Journal of Physics communications, 2(10), 105016. https://doi.org/10.1088/2399-6528/aae7e6

Guy, T. T., \& Bogning, J. R. (2018). Solitary wave solutions of modified telegraphist equations modeled in an electrical line. Physics Journal, 4(3), 29-36.

Njikue, R., Bogning, J. R., \& Kofane, T. C. (2018). Exact bright and dark solitary wave solutions of the generalized higher-order nonlinear Schrödinger equation describing the propagation of ultra-short pulse in optical fiber. Journal of Physics Communications, 2(2), 025030. https://doi.org/10.1088/2399-6528/aaaf3b

Njikue, R., Bogning, J. R., \& Kofané, T. C. (2018). Higher-Order Nonlinear Schrödinger Equation Family in Optical Fiber and Solitary Wave Solutions. American Journal of optics and photonics, 6(3), 31. https://doi.org/10.11648/j.ajop.20180603.12 
Sarwar, S., \& Rashidi, M. M. (2016). Approximate solution of two-term fractional-order diffusion, wave-diffusion, and telegraph models arising in mathematical physics using optimal homotopy asymptotic method. Waves in random and complex media, 26(3), 365-382. https://doi.org/10.1080/17455030.2016.1158436

Takongmo, G. T., \& Bogning, J. R. (2018). (kink, kink) and (pulse, pulse) exact solutions of equations modeled in a nonlinear capacitive electrical line with capacitor. American Journal of circuits, systems and signal processing. $4(3), 45-53$.

Tchinda, C. N., \& Bogning, J. R. (2020). Solitary Waves and Property Management of Nonlinear Dispersive and Flattened Optical Fiber. American Journal of Optics and Photonics, 8(1), 27-32. https://doi.org/10.11648/j.ajop.20200801.13

\section{Copyrights}

Copyright for this article is retained by the author(s), with first publication rights granted to the journal.

This is an open-access article distributed under the terms and conditions of the Creative Commons Attribution license (http://creativecommons.org/licenses/by/4.0/). 